\title{
On the search for elements beyond $Z=118$. An outlook based on lessons from the heaviest known elements
}

\author{
Christoph E. Düllmann ${ }^{1,2,3,}$ \\ ${ }^{1}$ Institute of Nuclear Chemistry, Johannes Gutenberg University, 55128 Mainz, Germany \\ ${ }^{2}$ GSI Helmholtzzentrum für Schwerionenforschung, 64291 Darmstadt, Germany \\ ${ }^{3}$ Helmholtz Institute Mainz, 55099 Mainz, Germany
}

\begin{abstract}
Recently, IUPAC approved all elements up to $Z=118$ as discovered. Search experiments for the heavier elements with $Z=119$ and 120 have been performed in recent years, but have so far not led to their discovery. I will review some aspects associated with the study and identification of the heaviest known elements that are relevant for future search experiments for elements beyond $Z=118$ and highlight pressing issues that should be addressed, both on the experimental as well as on the theory side, to allow for performing these future experiments under improved and better informed conditions.
\end{abstract}

\section{Introduction}

At the time of the Nobel Symposium NS160, held in beautiful Bäckaskog Castle, Sweden, in May/June 2016, all elements up to $Z=118$ have been officially accepted by IUPAC as discovered $[1,2]$. One week later, the recommended names for the four most recent elements were announced. Figure 1 shows the corresponding cut-out of the chart of nuclei (with decay properties updated according to [3]), where all known or claimed nuclei are indicated in coloured boxes, overlaying the calculated shell-correction energy landscape according to a macroscopic-microscopic model [4]. Also indicated (by white boxes for yet unknown isotopes) are the hypothetical decay chains originating from the $3 n$ and $4 n$ compound nucleus (CN) evaporation channels following complete fusion of ${ }^{50} \mathrm{Ti}+{ }^{249} \mathrm{Bk}$ leading to ${ }^{299} 119^{*}$ (red bordered boxes), ${ }^{50} \mathrm{Ti}+{ }^{249} \mathrm{Cf}$ leading to ${ }^{299} 120^{*}$ (light blue bordered boxes), and ${ }^{54} \mathrm{Cr}$ $+{ }^{248} \mathrm{Cm},{ }^{58} \mathrm{Fe}+{ }^{244} \mathrm{Pu}$, and ${ }^{64} \mathrm{Ni}+{ }^{238} \mathrm{U}$, which all lead to ${ }^{302} 120 *$ (dark blue bordered boxes). Decay modes of unknown isotopes were assumed based on [5-7].

On the first day of this Nobel Symposium, the introductory speaker for the physics of the superheavy elements, Matti Leino, listed among his conclusions that it is "difficult to increase $Z$, and $N$. How about deepening our understanding of already synthesized species?" I follow this recipe and devote Sect. 2 to lessons from known elements that bear relevance to the search for new elements. Section 3 will be devoted to an outlook, going beyond $Z=118$, picking up on the reactions mentioned above.

\footnotetext{
a e-mail: duellmann@uni-mainz.de
} 


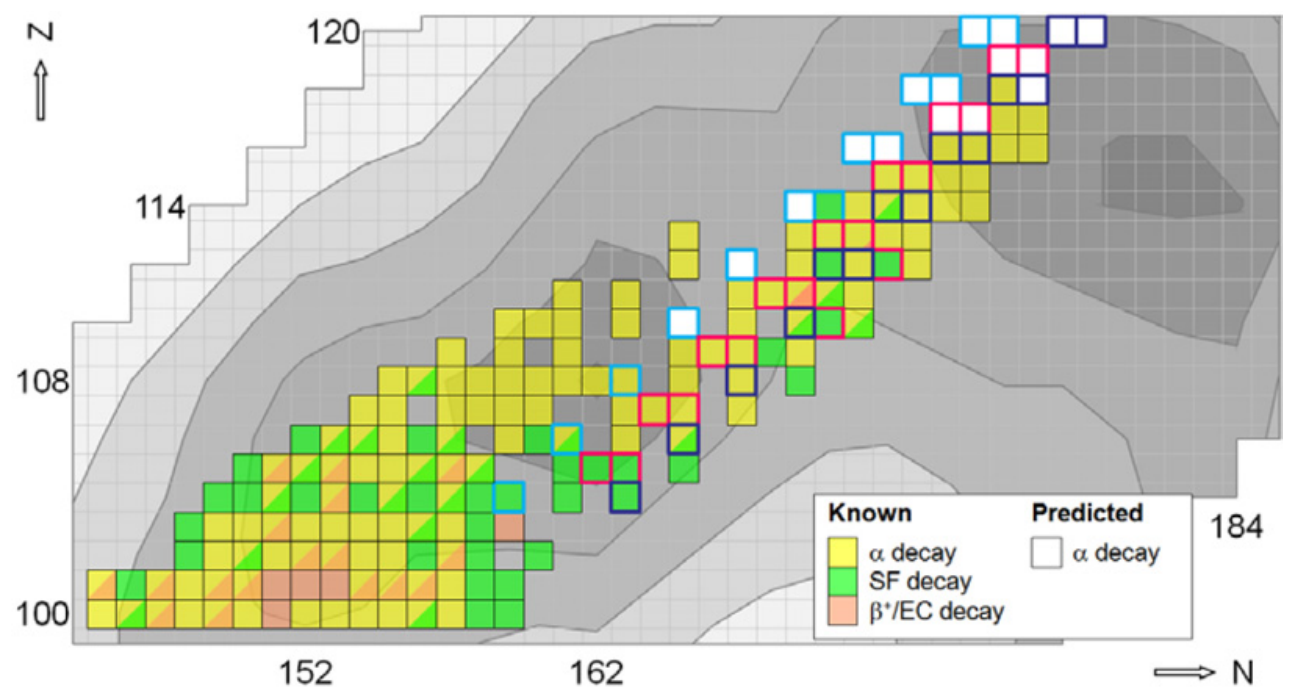

Figure 1. Chart of nuclei of the heaviest elements. The underlying contour plot shows calculated shellcorrection energies [4]. Known nuclei are indicated by coloured boxes, new nuclei occurring in the hypothetical $3 n$ and $4 n$ decay chains produced in complete fusion of different target and projectile combinations leading to $Z=119$ and $Z=120$ are indicated by white boxes. See text for more details.

\section{Lessons from known elements}

For this, I will look at the case of the element 115. In experiments performed at the Flerov Laboratory for Nuclear Reactions (FLNR) in Dubna, Russia [8, 9], at the GSI Helmholtzzentrum in Darmstadt, Germany $[3,10]$, and at the Lawrence Berkeley National Laboratory (LBNL) in Berkeley, CA, USA [11], over 100 decay chains have been observed following the irradiation of ${ }^{243} \mathrm{Am}$ targets with ${ }^{48} \mathrm{Ca}$ beams and subsequent separation of evaporation residues in gas-filled recoil separators. All were assigned to originate from element 115, produced in complete fusion, neutron-evaporation reactions. Indirect arguments on the reaction mechanism and nuclear structure of the produced nuclei were invoked to support this assignment [8, 9], along with the IUPAC-preferred tool of "crossbombardments", in which a certain nucleus is produced in multiple pathways; for example, in the case of element 115 , the claimed production of ${ }^{289} 115$ via ${ }^{243} \mathrm{Am}\left({ }^{48} \mathrm{Ca}, 2 n\right)$ and via ${ }^{249} \mathrm{Bk}\left({ }^{48} \mathrm{Ca}, 4 n\right){ }^{293} 117 \rightarrow \alpha$ decay [1]. None of the experiments performed to date were able to directly identify the $Z$ or the $A$ of any nucleus ascribed to belong to any element 115 decay chain (or, in a broader perspective, of any nucleus produced in a ${ }^{48} \mathrm{Ca}+$ actinide target reaction). While the current assignment appears correct based on indirect arguments, K.E. Gregorich argues in his contribution to these proceedings that the assignment appears "good, but not good enough" [12]. Still, IUPAC approved these data as originating from element 115 [1], and especially highlighted the cross-bombardment aspect in its assessment, which formed the basis for their approval. The IUPAC-approved cross bombardment case of elements 115 and 117, originally invoked by the FLNR-led collaborations has since been challenged [13], and the identification of scientifically sound connections of element 117 and element 115 chains is ongoing [14]; even if found, they will be unable to prove the $Z$ of the originating element, though. The safe, proven assignment of the $\alpha$ decay of the first member of any decay chain to element 115 is only possible if the nuclear reaction can be shown to lead to an isotope of element 115 (i.e., if complete fusion is achieved, followed by 


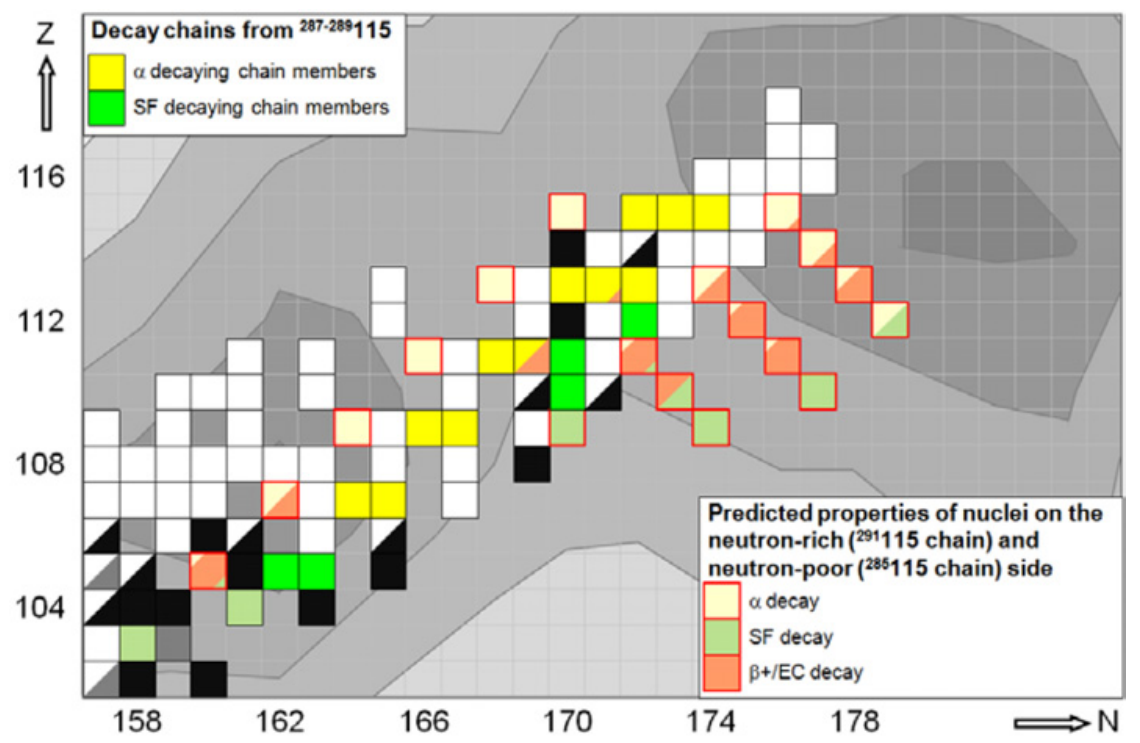

Figure 2. Black-outlined coloured boxes: decay chains populated in ${ }^{243} \mathrm{Am}\left({ }^{48} \mathrm{Ca}, 2-4 n\right)$ reactions, as given in "scenario 2" in [3]. White (black) boxes: other known $\alpha$-decaying (SF-decaying) nuclei. Redbordered coloured boxes: predicted decay properties [16] of element 115 decay chains located on the neutron-rich $\left({ }^{291} 115\right)$ and neutron-deficient $\left({ }^{285} 115\right)$ side of those from the ${ }^{243} \mathrm{Am}\left({ }^{48} \mathrm{Ca}, 2-4 n\right)$ reactions. The endpoints of the ${ }^{285} 115$ chain $\left({ }^{261} \mathrm{Lr}\right.$ and $\left.{ }^{265} \mathrm{Rf}\right)$ are known and decay by SF.

the evaporation exclusively of neutrons), and if its decay is uniquely detected. I will focus here on the second aspect. For this, it is illustrative to review predictions on partial half-lives of different decay modes in the relevant region of the nuclear chart. The growing importance of $\beta^{+}$/electron capture (EC) decay, especially towards the neutron-rich region in the isotopic chains of element 113 and 115, has been noted, e.g., in [15, 16], see Fig. 2.

In none of the presently reported decay chains assigned to proceed through element 115 are there members that were originally (neither by IUPAC) assigned to possess a $\beta^{+} / \mathrm{EC}$ branching. To fully appreciate the relevance of this, one should bear in mind that the detection techniques used for the study of the ${ }^{48} \mathrm{Ca}+{ }^{243} \mathrm{Am}$ reaction at the recoil separators in Dubna, Darmstadt, and Berkeley, are sensitive to $\alpha$ as well as to spontaneous fission (SF) decay; however, the direct detection of EC decay is not possible. The presence of an EC-decaying member in the chain could only be inferred indirectly by comparing decay properties of later chain members with known data - which is only possible if decay properties of $(i)$ nuclei populated if EC decays are present, as well as of (ii) those populated in the absence of EC decay, are known. None of the nuclei populated by the decay chains obtained in the ${ }^{48} \mathrm{Ca}+{ }^{243} \mathrm{Am}$ reaction have been known before, though.

Chemical approaches able to separate individual elements have the power to achieve $Z$ identification. For this, chemical properties of the element need to be established and exploited. The heaviest element, which had its chemical properties reproducibly studied, is $\mathrm{Cn}(Z=112)$ using the isotope ${ }^{283} \mathrm{Cn}$ produced as $\alpha$-decay daughter in the reaction ${ }^{242} \mathrm{Pu}\left({ }^{48} \mathrm{Ca}, 3 \mathrm{n}\right){ }^{287} \mathrm{Fl}$, see review in [17]. $Z$ identification is still not fully direct, but relies on chemical similarities with the lighter members in group 12 of the Periodic Table [18]. For element 113, first attempts for its chemical study have been performed, and for Fl, conflicting results on its chemical behaviour are reported [19, 20], see discussion in [17]. The power of chemical separators can only be fully employed, if the chemical properties of the element to 
be studied are already established. Once this is achieved, chemical studies of isotopes with unknown $Z$ will allow verifying its atomic number.

Coming back to the decay chains allegedly originating from element 115 , more specifically to the 96 five- $\alpha$ long chains from ${ }^{288} 115$, we note that there are arguments supporting that no chain member is present which decays (exclusively or in large fractions) by an intermediate $\beta^{+} / \mathrm{EC}$ decay, which would render the chain to continue through eveneven isotopes: first, statistical analyses of lifetime distributions of each individual generation within these chains showed these distributions to be consistent with what would be expected if every specific decay step occurred from the same nuclear state in all chains. Significant abundance of branched decay in any member is thus unlikely and would only go undetected by this method if a partially $\beta^{+} /$EC-decaying member with a lifetime that is very long or very short compared to that of its daughter were present. Only in these two extreme cases would the distribution of apparent lifetimes (which would then sometimes be the sum of lifetimes of $\beta^{+} / \mathrm{EC}$ decay and subsequent $\alpha$ decay) at this position in the decay chain fit with what is expected for a decay of a single nuclear state. Secondly, $\beta^{+} / \mathrm{EC}$ decay in this chain would populate isotopes known or expected to be fast fissioning even-even nuclei. Therefore, experimental evidence suggests that no decay branches other than $\alpha$ (and terminating SF, potentially preceded by $\beta^{+} /$EC decay) are present in these long chains.

For ${ }^{289} 115$, data is scarcer, precluding as definite statements. Interestingly, isotopes of elements 115,113 , and 111 that are only slightly more neutron-rich than those produced in the ${ }^{48} \mathrm{Ca}+{ }^{243} \mathrm{Am}$ reaction are predicted to possess sizable $\beta^{+} / \mathrm{EC}$ branches. As follows from Fig. 4 of [15], this decay mode is expected also in lighter isotopes of these elements, also in those populated in the decay chains of ${ }^{288,289} 115$. On the neutron-deficient side, taking the 285115 chain as an example, $\beta^{+} / \mathrm{EC}$ branches are furthermore predicted for the element 107 and 105 members. The $\alpha$ and $\beta^{+} / \mathrm{EC}$ daughters of the element 105 member, i.e., ${ }^{261} \mathrm{Lr}$ and ${ }^{265} \mathrm{Rf}$, are known to decay by SF, in agreement with the calculations. The question thus arises how safe a $Z$ assignments can be if $\beta^{+} / \mathrm{EC}$ decay as a prominent possibility is neglected (or, more accurately: not detectable). The problem is illustrated in Fig. 3. Due to the multiple decay modes with significant branches as they are predicted to occur in several of the populated nuclei (assuming decay properties as predicted in [16]) each decay chain from ${ }^{291} 115$ will pass through one out of in total 19 different pathways. In Fig. 3, just five out of these pathways are highlighted. All include two $\alpha$ decays and different numbers of $\beta^{+} / E C$ decaying members at different positions in the chains, and are terminated by SF. Table 1 presents these five pathways in more detail.

As can be seen, all five pathways lead to the detection of $\alpha-\alpha-$ SF decay chains in the absence of sensitivity to $\beta^{+} / E C$ decays. None of the three detected nuclei can be fixed in $A$ or $Z$. Focusing on the $Z$ of the first detected decaying nucleus, we see that this will be 115 in three of the five pathways, 114 in another one, and 113 in the last discussed one!

A safe assignment of the first $\alpha$ particle to element 115 is thus impossible. It thus appears crucial to expand current techniques to be sensitive also to $\beta$-decay processes. Detection of this decay mode and its detailed study are routinely performed in other scientific communities. As just one example, neutrino mass determination via a "missing-mass" type of approach, using EC decaying isotopes - e.g., ${ }^{163} \mathrm{Ho}$, which is used due to its very low $Q_{E C}$ value [21] - is mentioned, with the "Electron Capture in Holmium-163" (ECHo) collaboration $[22,23]$ being one of the groups working in this field. Metallic magnetic microcalorimeters [24] are used. In an exploratory study, similar detectors have been applied for studies of fusion-evaporation reaction products behind the SHIP separator at GSI [25], but the technique has not yet reached maturity to be directly applicable in superheavy element research. Approaches based on more traditional techniques may appear better suited to 


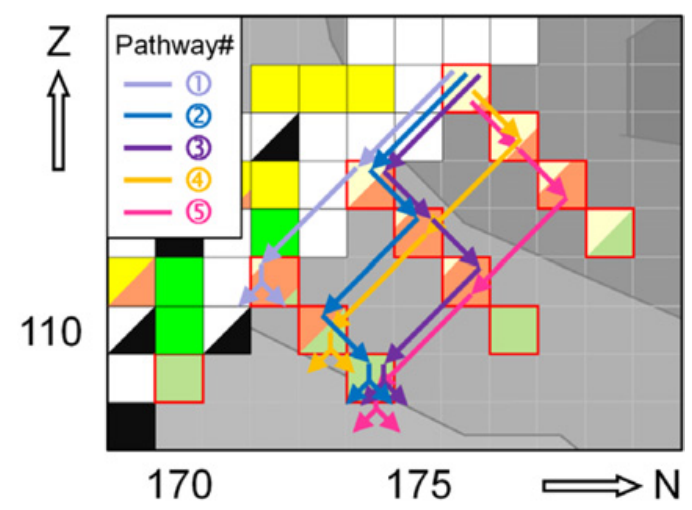

Figure 3. Cut-out from Fig. 2, indicating predicted decay modes and their relative importance for ${ }^{291} 115$ and its daughters. Among all possible 19 decay pathways, five are selected; see text for more details.

Table 1. Five decay pathways given in Fig. 3, originating from ${ }^{2} 91115$.

\begin{tabular}{|c|c|c|c|c|c|}
\hline$\#$ & Decay pathway & Apparent chain $^{1}$ & $\alpha_{1}$ & $\alpha_{2}$ & SF \\
\hline 1 & $\alpha-\alpha-$ SF & $\alpha-\alpha-$ SF & ${ }^{291} \mathbf{1 1 5}$ & ${ }^{287} 113$ & ${ }^{283} \mathbf{1 1 1}$ \\
\hline 2 & $\alpha-\beta^{+} / \mathrm{EC}-\alpha-\beta^{+} / \mathrm{EC}-\mathrm{SF}$ & $\alpha-\alpha-\mathrm{SF}$ & ${ }^{291} \mathbf{1 1 5}$ & ${ }^{287} \mathbf{1 1 2}$ & ${ }^{283} \mathbf{1 0 9}$ \\
\hline 3 & $\alpha-\beta^{+} / \mathrm{EC}-\beta^{+} / \mathrm{EC}-\alpha-\mathrm{SF}$ & $\alpha-\alpha-\mathrm{SF}$ & ${ }^{291} \mathbf{1 1 5}$ & ${ }^{287} \mathbf{1 1 1}$ & ${ }^{283} \mathbf{1 0 9}$ \\
\hline 4 & $\beta^{+} / \mathrm{EC}-\alpha-\alpha-\mathrm{SF}$ & $\alpha-\alpha-\mathrm{SF}$ & ${ }^{291} \mathbf{1 1 4}$ & ${ }^{287} \mathbf{1 1 2}$ & ${ }^{283} \mathbf{1 1 0}$ \\
\hline 5 & $\beta^{+} / \mathrm{EC}-\beta^{+} / \mathrm{EC}-\alpha-\alpha-\mathrm{SF}$ & $\alpha-\alpha-\mathrm{SF}$ & ${ }^{291} \mathbf{1 1 3}$ & ${ }^{287} \mathbf{1 1 1}$ & ${ }^{283} \mathbf{1 0 9}$ \\
\hline
\end{tabular}

${ }^{1}$ In case of non-detection of $\beta^{+} / \mathrm{EC}$ decay.

achieve direct EC observation [26], including delayed $X$-ray coincidence observation, which is possible if the detection system is operated in a low-background environment as present, e.g., at the SHIP [27] or the planned BGS-FIONA setup [28].

Having discussed $Z$ assignment and the problem of detecting $\beta^{+} /$EC-decaying isotopes, it is worthwhile to discuss the assignment of $A$ to observed decay chains. This is discussed in $[3,13,14]$ for chains produced in ${ }^{48} \mathrm{Ca}+{ }^{243} \mathrm{Am}$ reactions. Important aspects include that nuclei having significant branches for different decay modes are prevalent also among the heaviest known elements. Therefore, the length of a decay chain (i.e., the number of chain members) cannot be used to judge which set of chains originates from the same nucleus. Not even elaborate statistical analyses as presented in [3] are sufficient to judge whether the $A$ of one type of chain is identical or different to that of a distinctly different set of chains, not least because the occurrence of isomeric states, which decay, e.g., by $\alpha$ decay to analog states in the daughter rather than to their own ground state, is expected according to theoretical calculations (see Sect. 6 in [3]).

To conclude, despite all progress made in recent years, culminating in the IUPACapproved discovery and naming of all elements up to $Z=118$, the direct identification of $A$ or $Z$ of any nucleus produced in ${ }^{48} \mathrm{Ca}+$ actinide reactions has not been achieved yet. Detection to date relies exclusively on the observation of $\alpha$ and SF decay. $\beta^{+} / \mathrm{EC}$ decay, expected to be present also in the region that is already being explored, cannot be detected experimentally. 
Table 2. Fusion reactions employed to search for elements beyond $Z=118$.

\begin{tabular}{|l|c|c|c|c|}
\hline & $\begin{array}{c}\text { Compound } \\
\text { nucleus }\end{array}$ & $\begin{array}{c}\text { Beamtime } \\
\text { invested }\end{array}$ & $\begin{array}{c}\text { Cross } \\
\text { section } \\
\text { limit }^{1}\end{array}$ & Ref. \\
\hline${ }^{50} \mathrm{Ti}+{ }^{249} \mathrm{Bk}$ & ${ }^{299} 119^{*}$ & 4.5 months & 2 & {$[30]$} \\
\hline${ }^{64} \mathrm{Ni}+{ }^{238} \mathrm{U}$ & ${ }^{302} 120^{*}$ & 4 months & $\geq 90 \mathrm{fb}$ & {$[31]$} \\
\hline${ }^{58} \mathrm{Fe}+{ }^{244} \mathrm{Pu}$ & ${ }^{302} 120^{*}$ & 2 months & $\geq 400 \mathrm{fb}$ & {$[32]$} \\
\hline${ }^{54} \mathrm{Cr}+{ }^{248} \mathrm{Cm}$ & ${ }^{302} 120^{*}$ & $>1$ month & $\geq 580 \mathrm{fb}$ & {$[33]$} \\
\hline${ }^{50} \mathrm{Ti}+{ }^{249} \mathrm{Cf}$ & ${ }^{299} 120^{*}$ & $>1$ month & 2 & {$[30]$} \\
\hline \\
${ }^{1} 63.2 \%$ confidence level ("one-event limit"). \\
${ }^{2}$ Under final analysis.
\end{tabular}

\section{The way to elements beyond $Z=118$}

Recent work suggests fusion reactions with actinide targets to provide the highest cross sections for the synthesis of elements beyond $Z=118$. To proceed on the successfully established ${ }^{48} \mathrm{Ca}$ beam route, targets beyond ${ }_{98} \mathrm{Cf}$ would be necessary. Current technology for the production of transuranium isotopes, e.g., at the ORNL's High Flux Isotope Reactor (HFIR) is capable of producing $\mu \mathrm{g}$ and pg amounts of long-lived ${ }_{99} \mathrm{Es}$ and ${ }_{100} \mathrm{Fm}$ isotopes, respectively [29]. This is orders of magnitude less than the experiments discovering and studying the heaviest known elements require. Thus, reactions induced by beams heavier than ${ }^{48} \mathrm{Ca}$ will be needed. The most commonly suggested reactions giving access to $Z \geq 119$ include ${ }^{48} \mathrm{Ca}+{ }^{249} \mathrm{Bk}$ (leading to $Z=119$ ) and ${ }^{64} \mathrm{Ni}+{ }^{238} \mathrm{U},{ }^{58} \mathrm{Fe}+{ }^{244} \mathrm{Pu},{ }^{54} \mathrm{Cr}+{ }^{248} \mathrm{Cm}$, and ${ }^{50} \mathrm{Ti}+{ }^{249} \mathrm{Cf}$ (all four leading to $Z=120$ ). All five have been employed in search experiments, but none has led to the conclusive discovery of a new element. 2 summarizes the efforts invested to date to find elements beyond $Z=118$.

As can be seen, more than one year of beamtime has been devoted to search experiments, which were carried out at GSI Darmstadt $\left({ }^{50} \mathrm{Ti}+{ }^{249} \mathrm{Bk},{ }^{64} \mathrm{Ni}+{ }^{238} \mathrm{U},{ }^{54} \mathrm{Cr}+{ }^{248} \mathrm{Cm}\right.$, and $\left.{ }^{50} \mathrm{Ti}+{ }^{249} \mathrm{Cf}\right)$ and FLNR Dubna $\left({ }^{58} \mathrm{Fe}+{ }^{244} \mathrm{Pu}\right)$. Most likely, non-observation of new elements was due to insufficient sensitivity. It is noteworthy that some of the predictions of the nuclear half-lives of the produced isotopes, especially in the case of $Z=120$, suggest these to be in the order of microseconds, i.e., comparable to the flight-time through the separator. Hence, losses due to decay-in-flight might have negatively affected these experiments. Preparations for new, improved experiments are under way at several laboratories. Thus, it appears appropriate to reflect on which aspects should get most attention to perform the best possible experiments, thus maximizing the likelihood of success. Obvious, purely technical aspects include higher primary beam intensities, targets that can withstand such intensities over extended periods $[34,35]$, more efficient separators, more sensitive detection systems that allow safe identification of single decay chains from new elements, and longer irradiation times. I will focus here on a different aspect: numerous theoretical calculations of maximum cross sections of the reactions presented in Table 2 have been published in recent years (see overview in [30] for the reactions leading to $Z=120$ ), which span ranges of several orders for each reaction. This translates to unknown minimum irradiation times needed to guarantee the discovery of a new element with high confidence. In general, maximum cross sections scale with reaction asymmetry, but theoretical uncertainties are comparable to differences between different reactions in many cases. ${ }^{54} \mathrm{Cr}+{ }^{248} \mathrm{Cm}$ is favoured among the reactions leading to ${ }^{302} 120^{*}$. The more asymmetric ${ }^{50} \mathrm{Ti}+{ }^{249} \mathrm{Cf}$ leads to ${ }^{299} 120^{*}$. The influence of the three extra neutrons in the former is often assumed to be favourable, as the reaction product is situated closer to the next spherical shell closure expected at $N=184$. The 


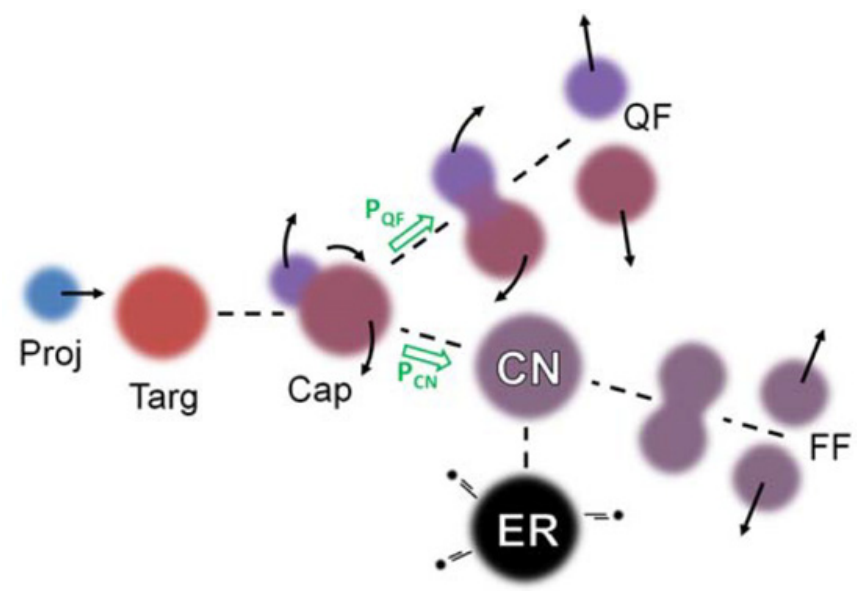

Figure 4. Schematic of the relevant processes in nucleus-nucleus collisions aimed at synthesizing the heaviest elements. Proj: projectile; Targ: target; Cap: captured system; CN: compound nucleus; ER: evaporation residue; QF: quasifission; FF: fusion-fission; $P_{C N}$ : probability for the captured system to form a compound nucleus; $P_{Q F}$ : probability for the captured system to fission. Obviously, $P_{Q F}=1-P_{C N}$.

opposite conclusion can also be reached, e.g., considering the fission barrier landscape as depicted in Fig. 6 of [36], which shows fission barrier heights calculated in the macroscopicmicroscopic approach, which is very successful in describing experimental data in the region of the heaviest known elements. Attempts to extract fission barrier heights from experimental observables have been performed [37] but are inherently model-dependent. Thus, no safe guidance on the optimum reaction, based purely on experimental data, exists.

Another aspect that is at least as critical as the proper choice of the nuclear reaction concerns the proper choice of the irradiation energy, as excitation functions are only a few $\mathrm{MeV}$ wide. Reliable experimental systematics exists for the ${ }^{48} \mathrm{Ca}+$ actinide reactions (e.g., Fig. 4 in [38]). However, the situation is dramatically different as soon as the reactions given in Table 2 are concerned. As detailed in [30], where the system ${ }^{50} \mathrm{Ti}+{ }^{249} \mathrm{Cf}$ is employed to illustrate the current situation, optimum beam energies suggested by different theoretical frameworks differ by more than $20 \mathrm{MeV}$ ! Recalling that typical energy ranges covered in a single experiment are roughly $5 \mathrm{MeV}$ highlights the need for a well-informed choice of the irradiation energy. Performing at multiple beam energies searches that are significantly more sensitive than the results shown in Table 2 (which were all obtained at one single energy) would consume years of beamtime. In this situation, performing complementary experiments studying these fusion reactions, which yield information that guides future new element search efforts, appears well justified. For this, we recall that the formation of a superheavy nucleus is parametrized as the product of three individual steps, each characterized with its own probability: $\sigma_{\mathrm{ER}}=\sigma_{\text {cap }} \cdot \mathrm{P}_{\mathrm{CN}} \cdot \mathrm{W}_{\text {sur }}$ with $\sigma_{E R}$ the evaporation residue (ER) cross section, $\sigma_{c a p}$ the capture cross section, $P_{C N}$ the compound nucleus formation probability, and $W_{\text {sur }}$ the exit channel survival probability. The formation of superheavy elements is significantly suppressed by the quasifission $(\mathrm{QF})$ process, in which two colliding nuclei reseparate on a fast time-scale before coalescing into a compound nucleus. See [39] for a detailed account, and Fig. 4 for a schematic of the relevant processes.

$\mathrm{QF}$ is the complementary process to compound nucleus formation, thus $P_{\mathrm{QF}}=1-P_{\mathrm{CN}}$, where $P_{\mathrm{QF}}$ can reach large values. In-depth studies of superheavy element formation reactions 
relying on ER observation are hampered by the tiny $\sigma_{\mathrm{ER}}$, which renders such studies very (beam)time consuming. The competing QF process profits from much higher rates for systems where $P_{\mathrm{CN}}$ is small. The understanding of $\mathrm{QF}$ processes has matured to a point where its detailed study, e.g., by recording mass angle distributions, provides significant insight. Thus, it appears timely to perform such studies of the reactions given in Table 2, and to gain an experimental data set of $P_{\mathrm{CN}}$ values, and of the heights of the Coulomb barrier in these reactions. Currently, theoretical frameworks only rarely predict observables of the QF process, but mostly focus on ER cross sections. Ideally, these frameworks will be extended to deliver predictions on observables of this "most likely outcome" of nucleus-nucleus collisions, i.e., QF (at least in cases where the underlying physics is contained in the framework). Such observables include mass angle distributions [40] or mass energy distributions [41]. Combined with the corresponding experimental data, which can be recorded in short experiments, this will allow testing different models, thus providing guidance concerning which ones deliver the most trustworthy predictions. Better informed selection of, e.g., the optimum irradiation energy will be invaluable. First steps in this direction have been made recently [39, 42]. A combined effort encompassing theoretical as well as experimental work will provide an improved basis for the certainly coming future efforts to discover new elements with atomic numbers $Z>118$ and to thus open a new row in the Periodic Table of the Elements.

\section{References}

[1] P.J. Karol et al., Pure Appl. Chem. 88, 139 (2016).

[2] P.J. Karol et al., Pure Appl. Chem. 88, 155 (2016).

[3] U. Forsberg et al., Nucl. Phys. A953, 117 (2016).

[4] Z. Patyk, R. Smolanczuk, A. Sobiczewski, Nucl. Phys. A626, 337c (1997).

[5] I. Muntian, Z. Patyk, A. Sobiczewski, Phys. At. Nucl. 66, 1015 (2003).

[6] I. Muntian et al., Acta Phys. Pol. B34, 2073 (2003).

[7] R. Smolańczuk, J. Skalski, A. Sobiczewski, Phys. Rev. C 52, 1871 (1995).

[8] Yu.Ts. Oganessian et al., Phys. Rev. C 69, 021601 (2004).

[9] Yu.Ts. Oganessian et al., Phys. Rev. C 87, 014302 (2013).

[10] D. Rudolph et al., Phys. Rev. Lett. 111, 112502 (2013).

[11] J.M. Gates et al., Phys. Rev. C 92, 021301(R) (2015).

[12] K.E. Gregorich, EPJ Web Conf., these proceedings (2016).

[13] U. Forsberg et al., Phys. Lett. B760, 293 (2016).

[14] D. Rudolph et al., (in preparation) (2016).

[15] A.V. Karpov et al., Int. J. Mod. Phys. E 21, 1250013 (2012).

[16] V.I. Zagrebaev, A.V. Karpov, W. Greiner, Phys. Rev. C 85, 014608 (2012).

[17] A. Türler, R. Eichler, A. Yakushev, Nucl. Phys. A944, 640 (2015).

[18] R. Eichler et al., Angew. Chem. Intl. Ed. 47, 3262 (2008).

[19] R. Eichler et al., Radiochim. Acta 98, 133 (2010).

[20] A. Yakushev et al., Inorg. Chem. 53, 1624 (2014).

[21] S. Eliseev et al., Phys. Rev. Lett. 115, 062501 (2015).

[22] L. Gastaldo et al., J. Low Temp. Phys. 176, 876 (2014).

[23] L. Gastaldo et al., Eur. Phys. J. Special Topics, (in preparation) (2016).

[24] L. Gastaldo et al., Nucl. Instrum. Methods A 711, 150 (2013).

[25] S. Kraft-Bermuth, PhD Thesis, Fachbereich Physik, JGU University Mainz, 2004.

[26] F.P. Heßberger et al., Eur. Phys. J. A 52, 192 (2016).

[27] F.P. Heßberger et al., Eur. Phys. J. A, (submitted) (2016). 
[28] J.M. Gates, EPJ Web Conf., these proceedings (2016).

[29] J.B. Roberto et al., Nucl. Phys. A944, 99 (2015).

[30] Ch.E. Düllmann et al., (in preparation) (2016).

[31] S. Hofmann, J. Phys. G 42, 114001 (2015).

[32] Yu.Ts. Oganessian et al., Phys. Rev. C 79, 024603 (2009).

[33] S. Hofmann et al., Eur. Phys. J. A 52, 180 (2016).

[34] S.N. Dmitriev and A.G. Popeko, J. Radioanal. Nucl. Chem. 305, 927 (2015).

[35] E. Jäger et al., J. Radioanal. Nucl. Chem. 299, 1073 (2014).

[36] M. Kowal, P. Jachimowicz, A. Sobiczewski, Phys. Rev. C 82, 014303 (2010).

[37] S. Hofmann et al., Eur. Phys. J. A 52, 116 (2016).

[38] Yu.Ts. Oganessian, and V.K. Utyonkov, Nucl. Phys. A944, 62 (2015).

[39] D.J. Hinde et al., EPJ Web Conf., these proceedings (2016).

[40] R. du Rietz et al., Phys. Rev. C 88, 054618 (2013).

[41] M.G. Itkis et al., Nucl. Phys. A944, 204 (2015).

[42] H.M. David et al., (in preparation) (2016). 